\title{
COMPARAÇÃO DE ORÇAMENTO DE OBRA DE SISTEMAS PREDIAIS COM E SEM UTILIZAÇÃO DE BIM ${ }^{1}$
}

\author{
BUDGETING BUILDING SYSTEMS WITH AND WITHOUT BIM: A \\ COMPARISON
}

\author{
Luís Felipe Guerretta \\ Universidade de São Paulo (USP) \\ Iguerretta@usp.br \\ Eduardo Toledo Santos \\ Universidade de São Paulo (USP) \\ etoledo@usp.br
}

\begin{abstract}
Resumo
O presente trabalho destina-se a compreender como a relação entre a construtora e subempreiteiros pode ser alterada por meio da implantação do processo BIM (Building Information Modeling) por parte da construtora, no que diz respeito à área de sistemas prediais. Esse trabalho, baseado em estudo de caso realizado em parceria com uma construtora, apresenta como esta utilizou o processo BIM para extração de quantitativos, bem como para realizar as equalizações das propostas recebidas dos subempreiteiros parceiros que trabalharam no processo tradicional de orçamentação. Os resultados sugerem que a forma de contratação destes subempreiteiros pode ser alterada pela construtora, reduzindo seus custos e, por consequência, potencializando lucros.
\end{abstract}

Palavras-chave: BIM. Orçamentos. Sistemas prediais. Extração de Quantitativos

\begin{abstract}
The present study aims to understand how the relationship between the construction company and the subcontractor can be changed through BIM (Building Information Modeling) implementation by the contractor, in the building systems area. This work, based on a case study carried out in association with a general contractor, shows how the construction company applied the BIM process to extract the quantitative information and also presents data on the equalization of the proposals submitted by the subcontractors, who work using the traditional process. The result suggests that the hiring process done by the construction company can be changed in order to reduce costs and consequently leverage its profits.
\end{abstract}

Keywords: BIM. Budget. Building systems. Quantity take off

\section{INTRODUÇÃO}

$\mathrm{Na}$ construção civil, uma das abordagens para o projeto e execução de obras mais tradicionais é o modelo DBB (Design-Bid-Build), no qual a principal vantagem é uma licitação mais competitiva para alcançar o menor preço possível para o proprietário

\footnotetext{
${ }^{1}$ GUERRETTA, L. F.; SANTOS, E. T.; Comparação de orçamento de obra de sistemas prediais com e sem utilização de BIM. In: ENCONTRO BRASILEIRO DE TECNOLOGIA DE INFORMAÇÃO E COMUNICAÇÃO NA CONSTRUÇÃO, 7., 2015, Recife. Anais... Porto Alegre: ANTAC, 2015.
} 
(EASTMAN et al., 2011). No modelo DBB, o proprietário estabelece os objetivos do empreendimento em questão e contrata um arquiteto que desenvolve todos os requisitos para construção. Na sequência, o investidor contrata uma construtora que, por sua vez, contrata os agentes responsáveis pelas disciplinas de MEP a serem realizadas para funcionamento do empreendimento. O processo de construção do empreendimento então é fragmentado entre diversos agentes e depende de formas de comunicação entre todos. Predominantemente, no Brasil, a comunicação em projetos da construção civil é baseada em papel e/ou arquivos CAD 2D.

Destes documentos, onde encontram-se os desenhos e especificações, espera-se obter, de forma suficiente, informações para facilitar o processo de orçamentação da construção do empreendimento.

Possíveis erros ou omissões nesta documentação resultam em custos imprevistos, atrasos na entrega da obra, deficiência nas funcionalidades para qual o empreendimento foi projetado e, em casos extremos, litígios entre os agentes envolvidos no processo (EASTMAN et al., 2011).

Todos os empreendimentos da construção civil começam com uma estimativa do seu custo. Portanto, uma das tarefas mais importantes no ramo da construção civil é o orçamento (KIM et al., 2006). De partida, sem um bom orçamento, será desafiador para o empreiteiro evitar prejuízos no contrato, independente do planejamento realizado por este.

Pode-se definir o orçamento como o processo técnico que se compromete a avaliar e prever o custo total para prestação de um serviço em determinado período de tempo, utilizando todas as informações disponíveis nos documentos do projeto e os recursos neste a aplicar.

O levantamento de quantitativos, tradicionalmente, é realizado manualmente, com auxilio de software para geração de planilha eletrônica disponível para o orçamentista. O método de quantificação dos insumos inclui a medição de todos os elementos do empreendimento, utilizando-se da escala em projetos impressos. Este método tende a ser tedioso e moroso, especialmente as transferências de medições para a planilha eletrônica, sendo que estas devem ser verificadas cuidadosamente para assegurar a exatidão (ALDER, 2006).

Este processo está sujeito a erro humano que, se não identificado, tende a propagar imprecisões relevantes no orçamento.

Estudos mostram que esse processo pode exigir de $50 \%$ a $80 \%$ do tempo de uma estimativa de custos em um projeto (SABOL, 2008).

Uma proposta para execução de determinado serviço é a soma de todos os preços unitários com uma margem de contribuição, onde a margem de contribuição compreende os encargos não industriais e uma margem líquida. Porém, é de extrema importância no processo de orçamento saber lidar com fatores, sejam eles internos ou externos ao processo, que influenciam na orçamentação, algo que não tem sido alvo de muita atenção. (AKINTOYE, 2000).

O objetivo principal de um orçamento é prever os custos do projeto o mais próximo possível da realidade, evitando assim duas situações prejudiciais para empresa: a sobreorçamentação e a sub-orçamentação. Uma sobreorçamentação pode culminar com a perda da concorrência almejada pelo empreiteiro. A sub-orçamentação pode causar prejuízos significativos para o empreiteiro.

Recomenda-se que o processo de gerenciamento destes fatores seja iniciado logo nas primeiras fases do orçamento, antes de começar fisicamente a obra e, preferencialmente, antes de assumir compromissos de prazo e custo com o cliente (DIEPENBRUCK, 2007). 
O objetivo deste trabalho é analisar, por meio de estudo de caso, como a construção do modelo BIM dos projetos de MEP pode ser utilizado para inovar no processo de orçamentação, comunicação entre os agentes envolvidos e execução de obras numa construtora.

Dentro deste contexto, pretende-se utilizar o processo BIM como meio de alcançar benefícios como:

- Extração de quantitativos dos elementos de MEP para orçamento;

- Análise de interferências e compatibilização de projetos virtualmente, antes do início da obra;

- Tomada de decisões mais criteriosa e menos subjetiva;

- Demonstração da responsabilidade da empresa construtora com o cliente;

- Aperfeiçoamento da comunicação e da experiência profissional entre a construtora, os subcontratados e o cliente.

\section{ORÇAMENTO DE SISTEMAS PREDIAIS PELA CONSTRUTORA}

A construtora não quantifica os componentes de sistemas prediais da obra pois seu foco é quantificar os insumos da parte civil do empreendimento. Em sua proposta é considerada uma verba para execução dessas disciplinas complementares, baseada na menor cotação entre os subempreiteiros parceiros.

No início do período de concorrência, a construtora recebe, do cliente ou gerenciador, todos os projetos referentes à obra para realizar seu orçamento. O primeiro passo tomado pela construtora é separar os projetos de sistemas prediais (elétrica, telefonia, automação, hidráulica, combate a incêndio e gás), e encaminhá-los para os subempreiteiros parceiros (instaladoras), para que estes realizem o orçamento destas disciplinas. Com o retorno da proposta de cada um desses parceiros, o orçamentista da construtora gera um mapa de cotações, demonstrando os valores, prazos e o escopo considerado por cada um desses parceiros.

Esse processo de orçamentação dos subempreiteiros, via de regra, deve ser realizado com extrema agilidade, pois o prazo da construtora para entrega da proposta para o cliente final usualmente varia de cinco a sete dias.

Dado que, geralmente, a realização da equalização das propostas orçamentárias de sistemas prediais é uma tarefa complexa pela grande variação de quantitativos indicados por cada empreiteiro, construtoras têm recorrido à modelagem BIM dos sistemas prediais pela necessidade de rastreamento e controle dos orçamentos recebidos dos subempreiteiros.

\section{ESTUDO DE CASO: MODELAGEM BIM PELA CONSTRUTORA}

A construtora, foco deste estudo de caso, conta com 27 anos de atuação no mercado da construção civil. É uma construtora de médio porte, com sede localizada no estado de São Paulo, realizando obras em diversos segmentos da construção, tendo mais de 2.000 obras concluídas nas áreas de edificações, corporativa, residenciais de alto padrão e prediais.

Esta construtora iniciou a implantação do processo BIM no ano de 2013. A proposta do departamento BIM é trabalhar em conjunto com o departamento de orçamentos, modelando toda parte civil e arquitetônica dos empreendimentos, para dar maior confiabilidade e reduzir os prazos de levantamentos desses quantitativos. 
Esse processo de modelagem implantado incentivou para que, no ano de 2015, a modelagem de sistemas prediais (MEP) acontecesse, dando a construtora o controle de todos os quantitativos do empreendimento a ser construído.

A equipe BIM de sistemas prediais foi formada por um engenheiro de instalações, com conhecimento do processo BIM, e um engenheiro de instalações que atuava em obras da empresa. Para validar o processo criado, no qual o departamento BIM de sistemas prediais auxiliará o departamento de orçamentos, uma obra foi escolhida como estudo de caso.

O projeto utilizado como estudo de caso é um centro comercial com $1500 \mathrm{~m}^{2}$ de área construída, 18 lojas e 36 vagas de estacionamento, localizado na cidade de Araraquara no estado de São Paulo, apresentado na Figura 1.

O prazo para entrega da proposta ao cliente final foi de sete dias úteis, tendo este projeto características similares a da maioria dos projetos realizados por esta construtora.

Figura 1 - Centro comercial, objeto do estudo de caso.

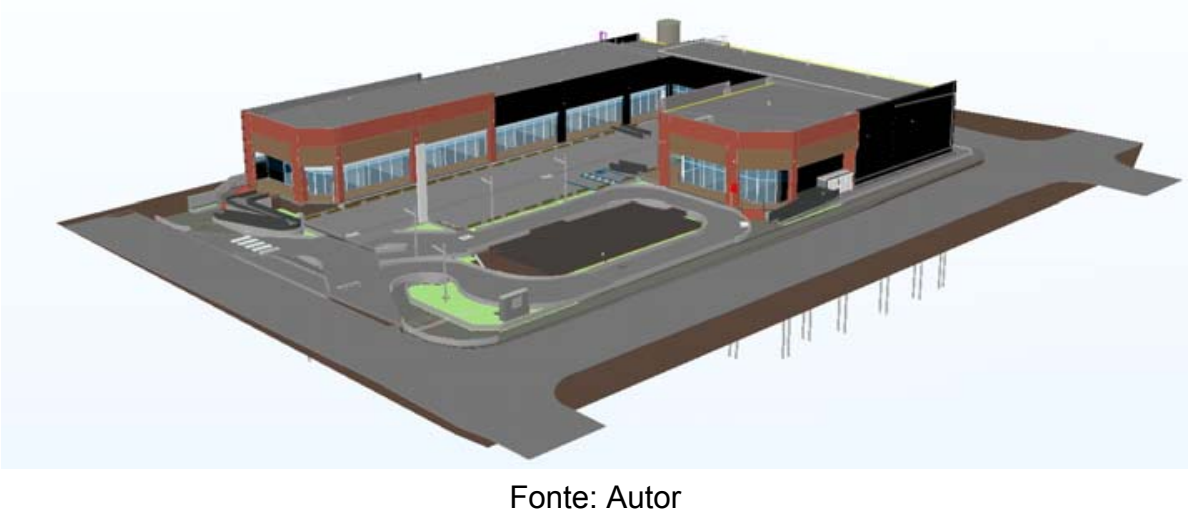

No primeiro dia de orçamentação, seguindo o processo tradicional, os projetos de sistemas prediais foram enviados para cinco subempreiteiros realizarem seus orçamentos. Em paralelo, a construtora passou para o departamento BIM todos os projetos do empreendimento.

A modelagem foi realizada com base nos projetos de sistemas prediais recebidos no início da concorrência da obra. Os componentes construtivos representados nos projetos 2D foram construídos em 3D com riqueza de detalhes adequada aos usos definidos pelo escopo de usos do modelo BIM desta pesquisa. Assim, os componentes foram desenvolvidos em L.O.D. 300 (Level of Development 300) (BIMFORUM, 2014), ponderadas as situações em que este nível foi inadequado ao uso do modelo BIM pretendido. Nestes casos, os componentes foram modelados em L.O.D. 400 (Level of Development 400).

A equipe BIM de arquitetura iniciou a modelagem dos projetos e, em três dias, o modelo BIM arquitetônico estava concluído. Esse modelo, então, foi disponibilizado para a equipe BIM de MEP iniciar seu trabalho.

Por decisão estratégica, para comparação entre propostas recebidas e a modelagem de MEP, o foco na modelagem foi dado, na disciplina elétrica, para extração de quantitativos de cabos, eletrodutos, eletrocalhas, perfilados e suas respectivas conexões e, na disciplina hidráulica, para tubulações e suas respectivas conexões. 
O modelo de MEP foi concluído também no prazo de três dias, como o modelo arquitetônico, e a Figura 2 mostra a perspectiva dos sistemas prediais do empreendimento modelado.

Após o processo modelagem, o primeiro relatório de interferências para compatibilização dos projetos foi gerado, identificando 148 pontos de interferências. Tais interferências levantadas se deram entre instalações $x$ instalações, instalações $x$ arquitetura, e instalações $x$ estrutura. Essas interferências, conforme mostrado na Figura 3, foram analisadas pelos engenheiros de instalações do departamento que relataram as melhores soluções de engenharia para os problemas identificados. Estas soluções então retornaram a equipe de modelagem de MEP para atualização do modelo, gerando assim a única revisão no processo.

Figura 2 - Perspectiva de parte dos sistemas prediais modelados.

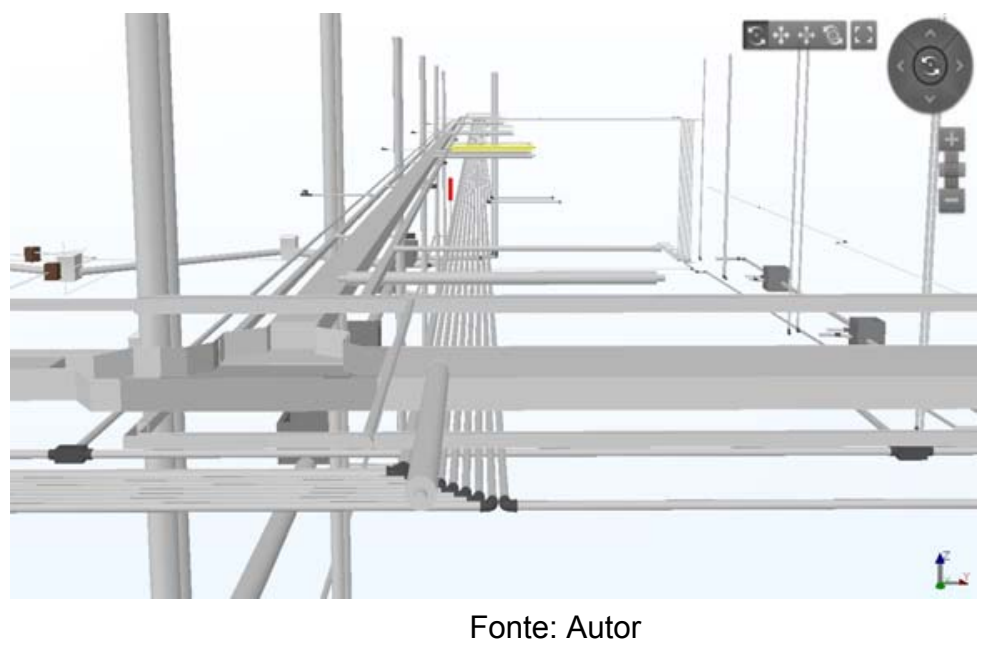

Figura 3 - Interferências detectadas no modelo BIM de sistemas prediais.

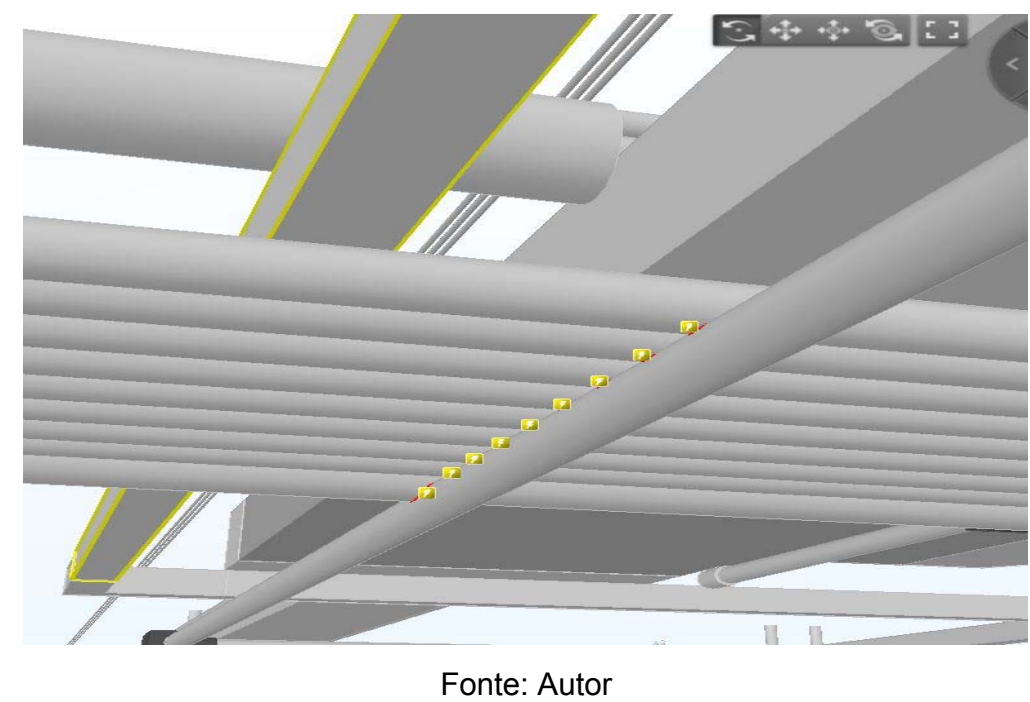

Após a validação do modelo pelo departamento de engenharia, a planilha de quantitativos foi extraída do mesmo de forma automática pelo software de modelagem. 
O sétimo dia do prazo de orçamentação foi utilizado para duas atividades distintas.

O departamento de suprimentos realizou o preenchimento da planilha de custos, baseandose nos quantitativos extraídos do modelo BIM. O departamento BIM, em conjunto com o departamento de orçamentos, realizou as equalizações dos quantitativos entre a planilha do modelo BIM e as propostas recebidas dos subempreiteiros. Dos cinco subempreiteiros acionados no início da orçamentação apenas três retornaram com suas propostas.

$\mathrm{Na}$ tabela de equalização, os materiais foram divididos em grupos, para facilitar a comparação.

O primeiro grupo da planilha é o de cabos, por ser o item de maior valor da obra na parte de sistemas prediais. Esse grupo foi dividido em quatro subgrupos; dentre os eles estão os cabos alimentadores, utilizados para ligação dos painéis elétricos da parte administrativa e a entrega do ponto de elétrica dos lojistas do empreendimento, conforme apresentado na Tabela 1. Esse subgrupo, em especifico, também foi quantificado pelo projetista, sendo sua tabela apresentada na prancha de alimentadores de elétrica do projeto em CAD 2D.

Tabela 1 - Equalização de quantitativos de cabos.

\begin{tabular}{|c|c|c|c|c|c|}
\hline DESCRIÇÃO & & & QUANTIDADES & & \\
\hline & PROJETO & $\begin{array}{l}\text { MODELO } \\
\text { BIM }\end{array}$ & $\begin{array}{l}\text { INSTALADORA } \\
\text { A }\end{array}$ & $\begin{array}{l}\text { INSTALADORA } \\
\text { B }\end{array}$ & $\underset{\mathrm{C}}{\text { INSTAL. }}$ \\
\hline \multicolumn{6}{|l|}{ GRUPO 1 - CABOS } \\
\hline \multicolumn{6}{|l|}{ CABOS ALIMENTADORES } \\
\hline $\begin{array}{l}\text { Cabo de Cobre Antichama Flexível - 750V } \\
\text { - Verde - 10,0mm2 }\end{array}$ & & & & & 210 \\
\hline $\begin{array}{l}\text { Cabo de Cobre Antichama Flexível - 750V } \\
\text { - Verde - 16,0mm2 }\end{array}$ & & & & & 140 \\
\hline $\begin{array}{l}\text { Cabo de Cobre Antichama Flexível - 750V } \\
\text { - Verde - 25,0mm2 }\end{array}$ & 368 & 320 & & & 20 \\
\hline $\begin{array}{l}\text { Cabo de Cobre Antichama Flexível - 750V } \\
\text { - Verde - 35,0mm2 }\end{array}$ & & & & & 490 \\
\hline $\begin{array}{l}\text { Cabo de Cobre Antichama Flexível - 750V } \\
\text { - Verde - 50,0mm2 }\end{array}$ & 356 & 318 & & & \\
\hline $\begin{array}{l}\text { Cabo de Cobre Antichama Flexível - 750V } \\
\text { - Verde - 70,0mm2 }\end{array}$ & 131 & 139 & & 10 & 35 \\
\hline $\begin{array}{l}\text { Cabo de Cobre Antichama Flexível - 750V } \\
\text { - Verde - 95,0mm2 }\end{array}$ & 35 & 40 & & & \\
\hline $\begin{array}{l}\text { Cabo de Cobre EPR } 1 \mathrm{Kv} \text { - Unipolar - } \\
\text { Preto - Afumex - } 10,0 \mathrm{~mm} 2\end{array}$ & & & & & 840 \\
\hline $\begin{array}{l}\text { Cabo de Cobre EPR } 1 \mathrm{Kv} \text { - Unipolar - } \\
\text { Preto - Afumex - } 16,0 \mathrm{~mm} 2\end{array}$ & & & & 492 & 280 \\
\hline $\begin{array}{l}\text { Cabo de Cobre EPR } 1 \mathrm{Kv} \text { - Unipolar - } \\
\text { Preto - Afumex - } 25,0 \mathrm{~mm} 2\end{array}$ & 1.104 & 960 & 2000 & 2412 & 360 \\
\hline $\begin{array}{l}\text { Cabo de Cobre EPR } 1 \mathrm{Kv} \text { - Unipolar - } \\
\text { Preto - Afumex - 35,0mm2 }\end{array}$ & & & & 216 & \\
\hline
\end{tabular}




\begin{tabular}{lccccc}
\hline \multicolumn{5}{c}{ DESCRIÇÃo } & \multicolumn{5}{c}{ QUANTIDADES } \\
\hline PROJETO & $\begin{array}{c}\text { MODELO } \\
\text { BIM }\end{array}$ & $\begin{array}{c}\text { INSTALADORA } \\
\text { A }\end{array}$ & $\begin{array}{c}\text { INSTALADORA } \\
\text { B }\end{array}$ & $\begin{array}{c}\text { INSTAL. } \\
\text { C }\end{array}$ \\
\hline $\begin{array}{l}\text { Cabo de Cobre EPR 1Kv - Unipolar - } \\
\text { Preto - Afumex - 50,0mm2 }\end{array}$ & 1.068 & 954 & 1550 & 1932 & 1430 \\
\hline $\begin{array}{l}\text { Cabo de Cobre EPR 1Kv - Unipolar - } \\
\text { Preto - Afumex - 70,0mm2 }\end{array}$ & 393 & 417 & 450 & 732 & 530 \\
\hline $\begin{array}{l}\text { Cabo de Cobre EPR 1Kv - Unipolar - } \\
\text { Preto - Afumex - 95,0mm2 }\end{array}$ & 105 & 120 & 250 & 192 & 140 \\
\hline TOTAL & $\mathbf{3 . 5 6 0}$ & $\mathbf{3 . 2 6 8}$ & $\mathbf{4 . 2 5 0}$ & $\mathbf{5 . 9 8 6}$ & $\mathbf{4 . 4 7 5}$ \\
\hline DIFERENÇA \% & $\mathbf{8 , 9 4 \%}$ & $\mathbf{0 , 0 0 \%}$ & $\mathbf{3 0 , 0 5 \%}$ & $\mathbf{8 3 , 1 7 \%}$ & $\mathbf{3 6 , 9 3 \%}$ \\
\hline
\end{tabular}

Nessa tabela percebemos duas situações críticas. A primeira é que as instaladoras não seguiram as especificações de materiais dada pelo projetista. $\mathrm{Na}$ modelagem BIM, seguimos rigorosamente as especificações técnicas listadas no projeto em CAD 2D (tipos de cabos). Na comparação com os dados do projetista, a diferença se deu apenas nos quantitativos, uma vez que no modelo BIM podemos garantir, com precisão, a distância percorrida por esses condutores. A segunda situação crítica fica pela diferença de quantidade de cabos quantificados entre todos os agentes. Para fins de comparação rápida, essa diferença foi mostrada na Tabela 1 em porcentagem da soma em metros dos cabos quantificados, ignorando a bitola dos mesmos.

Apresenta-se, na Tabela 2, o resumo dos principais itens de sistemas prediais, com os desvios de quantitativos entre a modelagem BIM e os apresentados pelos subempreiteiros. Essa tabela apresenta o resumo de totais, separando os itens por metro linear e as conexões em unidades, não considerando suas dimensões.

Tabela 2 - Resumo dos desvios em quantitativos.

\begin{tabular}{|c|c|c|c|c|}
\hline \multirow[t]{2}{*}{ DESCRIÇÃO } & \multicolumn{3}{|c|}{ QUANTIDADES } & \multirow[b]{2}{*}{ INSTALADORA C } \\
\hline & MODELO BIM & INSTALADORA A & INSTALADORA B & \\
\hline \multicolumn{5}{|l|}{ GRUPO 1 - CABOS (m) } \\
\hline TOTAL & 10.708 & 31.735 & 9.972 & 11.875 \\
\hline DIFERENÇA \% & $0,00 \%$ & $196,37 \%$ & $-6,87 \%$ & $10,90 \%$ \\
\hline \multicolumn{5}{|c|}{$\begin{array}{l}\text { GRUPO } 3 \text { - ELETRODUTOS DE } \\
\text { AÇO }(\mathrm{m})\end{array}$} \\
\hline TOTAL & 374 & 386 & 409 & 564 \\
\hline DIFERENÇA \% & $0,00 \%$ & $3,21 \%$ & $9,36 \%$ & $50,80 \%$ \\
\hline $\begin{array}{ll}\text { ELETRODUTOS } & \text { DE } \\
\text { CONEXÕES (pç) } & \\
\end{array}$ & AÇO & & & \\
\hline TOTAL & 274 & 0 & 0 & 0 \\
\hline
\end{tabular}




\section{TIC2015}

\begin{tabular}{|c|c|c|c|c|}
\hline \multirow[t]{2}{*}{ DESCRIÇÃO } & \multicolumn{3}{|c|}{ QUANTIDADES } & \multirow[b]{2}{*}{ INSTALADORA C } \\
\hline & MODELO BIM & INSTALADORA A & INSTALADORA B & \\
\hline DIFERENÇA \% & $0,00 \%$ & $-100,00 \%$ & $-100,00 \%$ & $-100,00 \%$ \\
\hline \multicolumn{5}{|l|}{$\begin{array}{l}\text { GRUPO } 6 \text { - ELETROCALHAS } \\
(\mathrm{m})\end{array}$} \\
\hline TOTAL & 140 & 147 & 183 & 220 \\
\hline DIFERENÇA \% & $0,00 \%$ & $5,00 \%$ & $30,71 \%$ & $57,14 \%$ \\
\hline $\begin{array}{l}\text { ELETROCALHAS CONEXÕES } \\
\text { (pç) }\end{array}$ & & & & \\
\hline TOTAL & 36 & 0 & 51 & 4 \\
\hline DIFERENÇA \% & $0,00 \%$ & $-100,00 \%$ & $41,67 \%$ & $-88,89 \%$ \\
\hline \multicolumn{5}{|l|}{$\begin{array}{l}\text { GRUPO } 8 \text { - TUBOS DE PVC } \\
\text { MARROM SOLDAVEL }(\mathrm{m})\end{array}$} \\
\hline TOTAL & 1.584 & 1.812 & 2.178 & 1.505 \\
\hline DIFERENÇA \% & $0,00 \%$ & $14,39 \%$ & $37,50 \%$ & $-4,99 \%$ \\
\hline \multicolumn{5}{|l|}{$\begin{array}{l}\text { CONEXÕES DE PVC MARROM } \\
\text { SOLDAVEL (pç) }\end{array}$} \\
\hline TOTAL & 241 & 0 & 121 & 375 \\
\hline DIFERENÇA \% & $0,00 \%$ & $-100,00 \%$ & $-49,79 \%$ & $55,60 \%$ \\
\hline \multicolumn{5}{|l|}{$\begin{array}{l}\text { GRUPO } 10 \text { - TUBOS DE PVC } \\
\text { TIPO SERIE R }(\mathrm{m})\end{array}$} \\
\hline TOTAL & 480 & 490 & 540 & 360 \\
\hline DIFERENÇA \% & $0,00 \%$ & $2,08 \%$ & $12,50 \%$ & $-25,00 \%$ \\
\hline $\begin{array}{llll}\text { CONEXÕES } & \text { DE } & \text { PVC } & \text { TIPO } \\
\text { SERIE R (pç) } & & & \\
\end{array}$ & & & & \\
\hline TOTAL & 241 & 0 & 216 & 0 \\
\hline DIFERENÇA \% & $0,00 \%$ & $-100,00 \%$ & $-10,37 \%$ & $-100,00 \%$ \\
\hline
\end{tabular}

\section{RESULTADOS}

Foram identificadas diferenças significativas entre os quantitativos levantados pelos subempreiteiros com relação ao levantamento realizado pela construtora via modelo BIM, conforme apresentado na Tabela 2. Esses elementos, considerados os itens " $A$ " da curva "ABC" de sistemas prediais, representaram a maior variação de quantitativos entre os orçamentos recebidos dos subempreiteiros, sendo que, em duas das três propostas, os subempreiteiros não quantificaram a maioria das conexões de sistemas prediais.

Com todos os quantitativos lançados na tabela de equalização, a construtora realizou outro estudo, comparando a diferença de custo entre comprar estes materiais de sistemas prediais e o valor destes materiais apresentados pelos instaladores na sua planilha orçamentária. Esse estudo, apresentado na Tabela 3, não contém a Instaladora C, pois, na 
apresentação da planilha orçamentária, essa empresa não separou os valores de material do valor de mão de obra, o que inviabilizou a comparação. Nesta tabela, os valores para a Construtora são referentes às quantidades levantadas através do modelo BIM.

Tabela 3 - Resumo de valores da equalização de valores de material.

\begin{tabular}{|c|c|c|c|c|c|c|}
\hline $\begin{array}{l}\text { Descrição } \\
\text { ELÉTRICA }\end{array}$ & \multicolumn{2}{|c|}{ Construtora } & \multicolumn{2}{|c|}{ Instaladora A } & \multicolumn{2}{|c|}{ Instaladora B } \\
\hline Cabos (BT / MT / Cobre Nu) & $\mathrm{R} \$$ & $74.175,50$ & $\mathrm{R} \$$ & $100.120,38$ & $\mathrm{R} \$$ & $169.437,62$ \\
\hline Eletrodutos e conexões de PVC roscável & $\mathrm{R} \$$ & $1.250,92$ & & & $\mathrm{R} \$$ & 537,40 \\
\hline Eletrodutos de aço & $\mathrm{R} \$$ & $4.889,20$ & $\mathrm{R} \$$ & $3.341,78$ & $\mathrm{R} \$$ & $3.676,00$ \\
\hline Kanaflex & $\mathrm{R} \$$ & $1.060,64$ & $\mathrm{R} \$$ & 537,53 & $\mathrm{R} \$$ & 557,00 \\
\hline Perfilados e acessórios galvanizados & $\mathrm{R} \$$ & $1.245,86$ & $\mathrm{R} \$$ & $2.020,59$ & $\mathrm{R} \$$ & $4.703,20$ \\
\hline Eletrocalhas e acessórios galvanizados & $\mathrm{R} \$$ & $2.646,68$ & $\mathrm{R} \$$ & $4.650,94$ & $\mathrm{R} \$$ & $21.388,08$ \\
\hline Conduletes e acessórios & $\mathrm{R} \$$ & 974,26 & $\mathrm{R} \$$ & 909,59 & $\mathrm{R} \$$ & 840,40 \\
\hline TOTAL ELÉTRICA & $\mathbf{R} \$$ & $86.243,06$ & $\mathbf{R} \$$ & $111.580,81$ & $\mathbf{R} \$$ & $201.139,70$ \\
\hline
\end{tabular}

\begin{tabular}{|c|c|c|c|c|c|c|}
\hline HIDRÁULICA & & & & & & \\
\hline Tubos e conexões de PVC marrom soldável & $\mathrm{R} \$$ & $6.548,65$ & $\mathrm{R} \$$ & $8.088,22$ & $\mathrm{R} \$$ & $12.001,92$ \\
\hline Tubos e conexões de PVC tipo esgoto & $\mathrm{R} \$$ & $2.916,73$ & & & & \\
\hline Tubos e conexões de PVC tipo Serie R & $\mathrm{R} \$$ & $20.691,04$ & $\mathrm{R} \$$ & $51.733,89$ & $\mathrm{R} \$$ & $22.495,80$ \\
\hline Tubos e conexões de cobre & $\mathrm{R} \$$ & $8.681,66$ & & & $\mathrm{R} \$$ & $16.540,80$ \\
\hline Tubos de aço carbono & $\mathrm{R} \$$ & $5.650,48$ & $\mathrm{R} \$$ & $16.753,87$ & $\mathrm{R} \$$ & $4.408,00$ \\
\hline $\begin{array}{l}\text { Conexões de ferro maleável classe } 10 \\
\text { galvanizada }\end{array}$ & $\mathrm{R} \$$ & $1.248,60$ & & & & \\
\hline TOTAL HIDRÁULICA & $\mathbf{R} \$$ & $45.737,16$ & $\mathbf{R} \$$ & $76.575,98$ & $\mathbf{R} \$$ & $55.446,52$ \\
\hline
\end{tabular}

\begin{tabular}{lccc}
\hline TOTAL GERAL (ELÈTRICA + HIDRÁULICA) & $R \$ 131.980,22$ & $R \$ 188.156,79$ & $R \$ 256.586,22$ \\
\hline DIFERENÇA - BASE MODELO BIM(\%) & $0,00 \%$ & $42,56 \%$ & $94,41 \%$ \\
\hline
\end{tabular}

$\mathrm{Na}$ Tabela 4, apresenta-se a diferença de valores para a compra de todos os materiais de sistemas prediais a serem utilizados neste empreendimento. Esses valores totais incluem os itens que não estavam na tabela de equalização, como, por exemplo, o transformador de potência ou a bomba de incêndio, os quais, mesmo tendo um alto valor individual, não apresentavam desvio na quantificação por terem apenas uma unidade alocada no projeto.

Tabela 4 - Resumo de custos totais de materiais.

\begin{tabular}{|c|c|c|c|c|c|c|}
\hline Descrição & & onstrutora & & staladora A & & staladora B \\
\hline Total de Materiais para Obra & $\mathrm{R} \$$ & $274.328,32$ & $\mathrm{R} \$$ & $416.622,57$ & $\mathrm{R} \$$ & $468.752,38$ \\
\hline Diferença & & $0,00 \%$ & & $51,87 \%$ & & $70,87 \%$ \\
\hline
\end{tabular}

Outro fator interessante de comparação é que, depois da equalização realizada, fica nítido os desvios de quantidades entre as instaladoras, mas ao comparar o valor final da proposta para realização da obra completa (material e mão de obra), todas apresentam valores muito próximos, conforme apresentado no mapa de cotação, Tabela 5. Isso evidencia uma metodologia de orçamentação por parte destes fornecedores que, aparentemente, não é diretamente correlacionada com os quantitativos do projeto. 
Tabela 5 - Resumo de totais para execução de sistemas prediais, no caso estudado.

\begin{tabular}{|c|c|c|c|c|}
\hline Descrição & Construtora & Instaladora A & Instaladora B & Instaladora C \\
\hline $\begin{array}{l}\text { Empreitada global } \\
\text { de instalações }\end{array}$ & $\mathrm{R} \$ \quad 479.328,32$ & $\mathrm{R} \$ \quad 1.056 .383,92$ & $\mathrm{R} \$ \quad 1.196 .493,80$ & $\mathrm{R} \$ \mathbf{1} \quad 1.120 .000,00$ \\
\hline Diferença \% & $0,00 \%$ & $120,39 \%$ & $149,62 \%$ & $133,66 \%$ \\
\hline
\end{tabular}

\section{CONCLUSÕES}

No modelo tradicional, para a construtora, todo o processo de orçamento de sistemas prediais terceirizado é subjetivo. A equalização das propostas é um processo moroso, e o prazo dado pelo cliente para entrega da proposta torna inviável um trabalho de detalhamento dessa equalização.

No estudo de caso realizado, ficou evidente o ganho de poder de negociação pela construtora no processo de orçamentação, uma vez que os custos do principal item de sua proposta estavam controlados e mensurados internamente.

A construtora ganhou agilidade no processo de coordenação e compatibilização de todos os projetos envolvidos na obra, uma vez que no modelo tradicional isso só ocorre no canteiro da obra, após a construtora ganhar a concorrência e mobilizar sua equipe. No processo proposto esse trabalho, a revisão da compatibilização de projetos foi realizada durante a modelagem, ainda na fase de orçamentação.

Como neste estudo de caso a precisão dos quantitativos foi garantida pela construtora via processo BIM, em vista dos resultados, mudou-se a forma de contratação do subempreiteiro de sistemas prediais. A construtora assumiu a responsabilidade de compra dos componentes de sistemas prediais e terceirizou apenas a mão de obra para execução dos serviços. Com essa alteração, a construtora economizou $\mathrm{R} \$ 577.055,60$ em relação ao custo se optasse pela instaladora com a proposta de menor valor.

O modelo BIM criado também foi utilizado na fase de contratação da empresa de mão de obra para visualização geral do empreendimento e apontamento dos pontos críticos e de possíveis interferências das instalações dos serviços a serem executados.

Outro ponto importante é a alteração da relação da construtora com a cadeia de suprimentos, uma vez que, com a necessidade da compra dos componentes de sistemas prediais, a construtora formou novas parcerias estratégicas com fornecedores para atender a nova demanda deste novo contrato e de todas as novas que virão a partir deste estudo de caso.

Entendemos que as subempreiteiras de sistemas prediais ainda não estão preocupadas ou preparadas para quantificar os insumos dos orçamentos que participam no prazo de que dispõem. Essas empresas usualmente consideram um valor de mercado por $\mathrm{m}^{2} \mathrm{de}$ construção, deixando qualquer detalhamento para ser realizado após ganhar a concorrência.

Vislumbra-se uma grande possibilidade de ganhos para as subempreiteiras de sistemas prediais que iniciarem a implantação do processo BIM. Não é o interesse da construtora assumir as atividades destas, mas a construtora pondera que a transparência na relação entre eles será fator determinante na retomada destas parcerias. 
As empresas de sistemas prediais terão um papel muito maior na cadeia construtiva considerando suas relações com a construtora e o investidor, uma vez que o processo BIM projeta que as empresas trabalhem de forma colaborativa e interoperável.

\section{AGRADECIMENTOS}

Os autores agradecem à construtora envolvida no estudo pelo fornecimento dos dados do caso apresentado. O segundo autor também agradece ao apoio do CNPq - Conselho Nacional de Desenvolvimento Científico e Tecnológico e da FAPESP - Fundação de Amparo à Pesquisa do Estado de São Paulo.

\section{REFERÊNCIAS}

AKINTOYE, A. Analysis of factors influencing project cost estimating practice. Construction Management \& Economics, v. 18, n. 1, p. 77-89, 2000.

ALDER, M. A. Comparing time and accuracy of building information modeling to onscreen take off for a quantity takeoff on a conceptual estimate. Dissertação (Master of Science). School of Technology Brigham Young University, 2006.

BIM FORUM Level of Development Specification - Version 2014 Issued: Dec. 30, 2014

DIEPENBRUCK, T. M. Gerenciamento de risco para obtenção da produtividade planejada. MBA em Tecnologia e Gestão na Produção de Edifício (Monografia). Universidade de São Paulo, 2007.

EASTMAN, C. et al. BIM handbook: A guide to building information modeling for owners, managers, designers, engineers and contractors. 2a. ed., Hoboken:John Wiley \& Sons, 2011.

$\mathrm{KIM}, \mathrm{H}$. et al. Generating construction schedules through automatic data extraction using open BIM (building information modeling) technology. Automation in Construction, v. 35, p. 285-295, 2013.

SABOL, L. Challenges in cost estimating with Building Information Modeling, 2008. Disponível em: < http://www.dcstrategies.net/files/2_sabol_cost_estimating.pdf>. Acesso em: 6 ago. 2015.

THOMPSON, P.; PERRY, J. G. (Ed.). Engineering construction risks: A guide to project risk analysis and assessment implications for project clients and project managers. Thomas Telford, 1992. 